\title{
Weight-based dosing in medication use: what should we know?
}

\author{
This article was published in the following Dove Press journal: \\ Patient Preference and Adherence \\ 12 April 2016 \\ Number of times this article has been viewed
}

\author{
Sheng-dong Pan' \\ Ling-ling Zhu \\ Meng Chen ${ }^{3}$ \\ Ping Xia' \\ Quan Zhou ${ }^{3}$ \\ 'Division of Medical Administration, \\ ${ }^{2}$ VIP Care Ward, Division of Nursing, \\ ${ }^{3}$ Department of Pharmacy, The \\ Second Affiliated Hospital, School \\ of Medicine, Zhejiang University, \\ Hangzhou, Zhejiang, People's Republic \\ of China
}

Background: Weight-based dosing strategy is still challenging due to poor awareness and adherence. It is necessary to let clinicians know of the latest developments in this respect and the correct circumstances in which weight-based dosing is of clinical relevance.

Methods: A literature search was conducted using PubMed.

Results: Clinical indications, physiological factors, and types of medication may determine the applicability of weight-based dosing. In some cases, the weight effect may be minimal or the proper dosage can only be determined when weight is combined with other factors. Medications within similar therapeutic or structural class (eg, anticoagulants, antitumor necrosis factor medications, P2Y12-receptor antagonists, and anti-epidermal growth factor receptor antibodies) may exhibit differences in requirements on weight-based dosing. In some cases, weight-based dosing is superior to currently recommended fixed-dose regimen in adult patients (eg, hydrocortisone, vancomycin, linezolid, and aprotinin). On the contrary, fixed dosing is noninferior to or even better than currently recommended weight-based regimen in adult patients in some cases (eg, cyclosporine microemulsion, recombinant activated Factor VII, and epoetin $\alpha$ ). Ideal body-weight-based dosing may be superior to the currently recommended total body-weightbased regimen (eg, atracurium and rocuronium). For dosing in pediatrics, whether weight-based dosing is better than body surface-area-based dosing is dependent on the particular medication (eg, methotrexate, prednisone, prednisolone, zidovudine, didanosine, growth hormone, and 13-cis-retinoic acid). Age-based dosing strategy is better than weight-based dosing in some cases (eg, intravenous busulfan and dalteparin). Dosing guided by pharmacogenetic testing did not show pharmacoeconomic advantage over weight-adjusted dosing of 6-mercaptopurine. The common viewpoint (ie, pediatric patients should be dosed on the basis of body weight) is not always correct. Effective weight-based dosing interventions include standardization of weight estimation, documentation and dosing determination, dosing chart, dosing protocol, order set, pharmacist participation, technological information, and educational measures.

Conclusion: Although dosing methods are specified in prescribing information for each drug and there are no principal pros and cons to be elaborated, this review of weight-based dosing strategy will enrich the knowledge of medication administration from the perspectives of safety, efficacy, and pharmacoeconomics, and will also provide research opportunities in clinical practice. Clinicians should be familiar with dosage and administration of the medication to be prescribed as well as the latest developments.

Keywords: body weight, dosage and administration, efficacy, medication safety, pediatrics, pharmacoeconomics

Department of Pharmacy, The Second Affiliated Hospital, School of Medicine, Zhejiang University, 88 jiefang Road, Shangcheng, Hangzhou 310009, Zhejiang, People's Republic of China

Tel +8657187784615

Fax +86 57I 87022776

Email zhouquan I42602@zju.edu.cn

\section{Introduction}

Generally, fixed-dosing regimen is potentially more convenient than weight-adjusted dosing regimen for both patients and clinicians. However, for the sake of safety and efficacy, weight-based dosing method is needed for particular population and also speci- 
fied in prescribing information for many medications. Joint Commission International (JCI) Accreditation Standards for Hospitals (5th edition) requires that the hospital policy should specify the weight-based dosing strategy in the particular circumstance (eg, pediatric patients, frail elderly patients). Clinicians should consider writing weight-adjusted orders whenever possible. ${ }^{1}$ Organizations must strive for strategies that achieve goals such as establishing processes for weighing and weight documentation, requiring pharmacy review of weight-based dosing regimens, ensuring sufficient and convenient availability of appropriate and properly functioning equipment for patient weighing and medication delivery, and using health information technology and clinical decision support software to promote safe and appropriate dosing. ${ }^{2}$

However, prescribing appropriate doses of drugs requiring weight-based dosing is still challenging due to poor awareness and adherence. A study showed that 3-12-yearold children with uncomplicated malaria were significantly more susceptible to receive wrong dose of artemetherlumefantrine, which should be prescribed on the basis of body weight. ${ }^{3}$ A retrospective cohort study of vancomycin prescribing in the emergency department (ED) showed that only $19.6 \%$ of patients received a consensus-guidelinerecommended dosing regimen $(15-20 \mathrm{mg} / \mathrm{kg}) .{ }^{4}$ A retrospective cost-savings analysis of imipenem/cilastatin in elderly patients revealed that only $37 \%$ of patient days of therapy was appropriate regarding dose and interval, and $\$ 11,500$ would be saved annually if clinicians could adjust dosage on the basis of body weight and creatinine clearance $(\mathrm{CrCl}){ }^{5}$ The manufacturer's recommended weight-adjusted dosing of tinzaparin could not warrant effective anticoagulation in the vast majority of pregnant women at high risk of venous thromboembolism (VTE) or diagnosed with acute VTE. ${ }^{6}$

Meanwhile, it has been recognized that drug plasma concentrations could be affected by multiple factors including dose, formulation form, route, frequency, administration time, drug-drug interaction, food-drug interaction, genetics, sex, age, body weight, pregnancy, circadian rhythms, comorbidities, pathophysiology status, and smoking. ${ }^{7}$ The effect of body weight on drug action may vary in extent. In some cases, weight can primarily determine the dosage; in other cases, the weight effect may be minimal or dosage can be affected only when weight is combined with other factors. Findings of sporadic studies in recent years indicated that fixed dosing was more advantageous than weight-based dosing in some cases, and also some key factors may challenge the necessity of weight-based dosing despite being specified in prescribing information. Therefore, it is necessary to let clinicians know the latest developments and the true circumstance in which weight-based dosing is of clinical relevance. Also, health care providers need to clearly understand the meaning of weight-based dosing requirements in the JCI Accreditation Standards for Hospital.

The Second Affiliated Hospital of Zhejiang University, School of Medicine, Zhejiang University, People's Republic of China (SAHZU) is a JCI-accredited academic medical center hospital. We have paid close attention to the knowledge of weight-based dosing during the preparation phase and in the postaccreditation era. Here we present a narrative review on this interesting topic.

\section{Methods}

Potentially relevant articles were identified by retrieving PubMed until February 2016. The search query was to identify each paper with the title containing a phrase like "weight-adjusted" or "weight-tailored" or "weight-based" or "weight-dependent" or "weight-independent", or two words "body weight and pharmacokinetics", with a filter of "species: human" and "languages: English". Two reviewers (SDP and $\mathrm{MC}$ ) independently retrieved the literature and screened the relevant studies. If they had a disagreement over including or excluding an article, the third reviewer (QZ) was consulted. Three hundred thirty-nine articles were identified. Forty-five records were excluded due to article types being letter, comment, editorial material, case report, review, meta-analysis, or newspaper article. After reviewing the titles of the remaining 294 articles, 204 records were not closely relevant to the topic, so they were directly excluded. Full-text articles were further assessed for eligibility. Regarding heparin, low-molecularweight heparins (LMWHs) and contrast, there were numerous studies with similar findings, and therefore only typical studies were included as examples. Six records were further excluded because they showed the advantage of weight-based dosing over fixed dosing, and such information has been specified in current prescribing information. Forty-six papers on weightbased dosing were finally chosen according to the inclusion/ exclusion criteria. Key information were summarized by data analysis and interpretation.

\section{Results}

\section{Factors affecting the applicability of weight-based dosing}

Clinical indications

Immunoglobulin G

For a specific medication, whether the dose should be weightadjusted may depend on clinical indications. Hodkinson et $\mathrm{al}^{8}$ 
compared the efficacy of immunoglobulin $\mathrm{G}(\mathrm{IgG})$ in obese and lean patients who received replacement or immunomodulatory therapy. Compared with lean patients, obese patients experienced higher serum trough $\operatorname{IgG}$ levels and $\mathrm{IgG}$ increments (but not IgG efficiencies) following a given weight-tailored dose $(P=0.001)$. Interestingly, this difference was observed only when IgG was prescribed for immunomodulation therapy rather than replacement therapy. It is promising to lower the dose in some obese patients receiving IgG for immunomodulation, which could save resources without compromising clinical outcome. ${ }^{8}$

\section{Nadroparin calcium}

Nadroparin calcium (Fraxiparine ${ }^{\circledR}$, Laboratoire GlaxoSmithKline, Marly-le-Roi Cedex, France) should be dosed by body weight for treatment of deep vein thrombosis (DVT), unstable angina, and non-Q-wave myocardial infarction. However, for another indication (ie, chemical prophylaxis of VTE), the dosing method of nadroparin calcium depends on clinical circumstances. For prevention of DVT or pulmonary embolism (PE) after general surgery, the adult dose of nadroparin is 2,850 anti-factor Xa IU injected subcutaneously once a day starting 2-4 hours before surgery and continuing for at least 1 week. However, for prevention of DVT or PE after hip replacement surgery, the adult dose of nadroparin is weight-adjusted before and after surgery. ${ }^{9}$

\section{Physiological factors \\ Enoxaparin}

A standard dose of enoxaparin (Lovenox ${ }^{\circledR}$; Sanofi-Aventis U.S. LLC, Bridgewater, NJ, USA) is frequently used for DVT prophylaxis, regardless of weight. ${ }^{10}$ Evidence suggests lower bioavailability of enoxaparin after a single daily subcutaneous dose $(40 \mathrm{mg})$ in critically ill patients with normal renal function when compared with medical patients in the normal ward. ${ }^{11}$ A study revealed that weight-based dosing of enoxaparin $(0.6 \mathrm{mg} / \mathrm{kg}$ twice daily) in trauma intensive care unit patients may provide superior VTE prophylaxis compared with standard dosing (30 mg twice daily). The change in enoxaparin dosing from $30 \mathrm{mg}$ to $0.6 \mathrm{mg} / \mathrm{kg}$ increased the percentage of therapeutic goal attainment in patients $(61 \%$ versus $8 \%, P<0.0001) .{ }^{12}$ Very recently, Chapman et $\mathrm{al}^{13}$ reported that standard non-weight-based dosing of enoxaparin for VTE prophylaxis could not reliably achieve target anti-factor Xa activity levels in trauma intensive care unit patients in contrast to weight-based manner of dosing ( $0.5 \mathrm{mg} / \mathrm{kg}$ subcutaneously every 12 hours). These studies may challenge the conventional viewpoint that it is unnecessary to excise weight-adjusted dosing of enoxaparin for VTE prophylaxis.

\section{Linezolid}

Linezolid injection $\left(Z Y V O X^{\circledR}\right.$, Pfizer Inc, New York, NY, USA) is indicated for treatment of infections caused by susceptible strains of microorganisms (eg, nosocomial pneumonia, community-acquired pneumonia, and vancomycin-resistant Enterococcus faecium infections). Intravenous or oral linezolid dose is $600 \mathrm{mg}$ every 12 hours for adults. The incidence rate of linezolid-induced thrombocytopenia varies, ranging from $1.9 \%$ to $17.1 \% .{ }^{14}$ Niwa et $\mathrm{al}^{15}$ examined whether the daily dose of linezolid in patients (age range, 33-85 years) based on the risk factors could affect the safety and efficacy of this antibacterial. Body weight less than $55 \mathrm{~kg}$ (odds ratio [OR]: $33.2, P=0.012$ ) and the baseline platelet count of $<200 \times 10^{3} / \mathrm{mm}^{3}$ (OR: $24.9, P=0.024$ ) were risk factors for thrombocytopenia induced by linezolid. For patients with one or two risk factors, weight-adjusted dosing of linezolid $(20 \mathrm{mg} / \mathrm{kg} / \mathrm{d})$ reduced the incidence of thrombocytopenia without compromising clinical efficacy (hazard ratio [HR]: $3.28, P=0.043) .{ }^{15}$ However, patients with neither of the two risk factors experienced no thrombocytopenia if they received standard daily dose of linezolid (1,200 mg).

\section{Vancomycin}

Maki et $\mathrm{al}^{16}$ elucidated the factors affecting vancomycin serum trough levels in Japanese adults. In patients with $\mathrm{CrCl}$ 30-50 $\mathrm{mL} / \mathrm{min}$, vancomycin dosing at $1 \mathrm{~g}$ daily had less opportunity for having trough levels over $20 \mu \mathrm{g} / \mathrm{mL}$, regardless of body weight. In patients with $\mathrm{CrCl}$ above $50 \mathrm{~mL} / \mathrm{min}$, the dosage of $2 \mathrm{~g} / \mathrm{d}$ was less likely to achieve trough levels below $10 \mu \mathrm{g} / \mathrm{mL}$ in patients under $55 \mathrm{~kg}$, rather than patients over $55 \mathrm{~kg}$. These data suggested that body weight and $\mathrm{CrCl}$ were two closely related physiological factors determining the optimal initial total daily dose of vancomycin. ${ }^{16}$

\section{Paricalcitol}

Paricalcitol injection (Zemplar ${ }^{\circledR}$, Hospira Inc, Lake Forest, IL, USA) is indicated for the prophylaxis and treatment of hyperparathyroidism secondary to stage 5 chronic kidney disease. The initial dose of Zemplar ${ }^{\circledR}$, ranging from 0.04 to $0.1 \mu \mathrm{g} / \mathrm{kg}$, is given as a bolus dose at appropriate time during dialysis. ${ }^{17} \mathrm{~A}$ randomized study compared the incidence of hypercalcemia in adult patients with end-stage renal disease on maintenance hemodialysis therapy following two regimens of paricalcitol (ie, one was based on baseline intact parathyroid hormone level and the other was tailored 
to body weight $[0.04 \mu \mathrm{g} / \mathrm{kg}])$. Paricalcitol treatment on the basis of degree of secondary hyperparathyroidism achieved good therapeutic outcomes with lower frequency of dose adjustments than weight-based dosing, without posing greater risk to hypercalcemia. ${ }^{18}$

\section{Warfarin}

The issue of warfarin dosage is a good example of multiple factors affecting the proper dose regimen. Using a multivariate linear regression analysis, Miao et $\mathrm{al}^{19}$ described the association of the warfarin dosage regimen in Chinese patients with variables such as genotypes of vitamin K epoxide reductase complex subunit 1 (VKORC1) and cytochrome P-450 2C9 (CYP2C9), age, body size, and body weight. Significant contributions to warfarin dose requirements were from age $\left(r^{2}=0.084 ; P<0.001\right)$, weight $\left(r^{2}=0.063\right.$; $P<0.001)$, VKORC1 genotype $\left(r^{2}=0.494 ; P<0.001\right)$, and four independent variables together (age + weight $+C Y P 2 C 9$ genotype + VKORC1 genotype $)\left(r^{2}=0.628\right.$; $P<0.001)$. Use of dosing regimens adjusted by age, weight, and genotypes of CYP2C9 and VKORC1 is beneficial to enhance safe use of warfarin. ${ }^{19}$ Yoo et $\mathrm{al}^{20,21}$ developed an age- and weight-based warfarin initiation nomogram for acute ischemic stroke patients and found that the age- and weight-based warfarin initiation nomogram could effectively predict appropriate warfarin dose due to shorter time to international normalized ratio (INR) $\geq 2.0$ ( $4.9 \pm 0.7$ versus $6.2 \pm 0.8$ days, $P=0.0008)$, more patients with therapeutic INR (2-3) at 5 days $(P=0.002)$, and lower risk of achieving INR $\geq 3.5(P=0.024)$ compared to physician-determined warfarin dosing.

\section{Isoniazid}

The usual oral dosage of isoniazid for adults is $5 \mathrm{mg} / \mathrm{kg}$ up to $300 \mathrm{mg}$ once daily or $15 \mathrm{mg} / \mathrm{kg}$ up to $900 \mathrm{mg} / \mathrm{d}$ twice or thrice a week. The use of personalized pharmacogeneticguided isoniazid dosage regimens that incorporate $N$-acetyltransferase 2 (NAT2) genotype and body weight may help to ensure achievement of therapeutic concentrations of isoniazid in tuberculosis patients. Based on multivariate stepwise linear regression analysis, a formula for predicting isoniazid concentration was as follows: isoniazid concentration $(\mathrm{mg} / \mathrm{L})=13.821-0.1 \times($ body weight, $\mathrm{kg})-2.273 \times$ (number of high activity alleles of NAT2; 0, 1,2). The modelbased treatment group experienced a higher frequency of falling in the therapeutic range of 3.0-6.0 $\mathrm{mg} / \mathrm{L}$ compared to the standard weight-based treatment group $(71.4 \%$ versus $40.0 \%, P=0.013) .^{22}$

\section{Local anesthetic}

Height- and weight-adjusted dosing and fixed-dosing were compared in terms of spinal anesthesia following administration of intrathecal hyperbaric bupivacaine during cesarean section. Although the median onset time of spinal block in fixed-dosing group was significantly faster than in adjusteddosing group, patients with fixed-dosing exhibited more frequent spinal block extended above T3 level (24\% versus $2 \% ; P<0.05)$ and suffered more frequent hypotension and more pronounced nausea and vomiting (64\% versus $30 \%$, $P<0.05)$ than those with adjusted-dosing. The height- and weight-adjusted dose use suitably restricted spinal block level for cesarean section, with a distinct advantage of significantly reduced dose, less hypotension, and with a similar neonatal outcome as fixed dose use. ${ }^{23}$ Harten et al ${ }^{24}$ compared two dosing regimens of local anesthetic for spinal anesthesia during elective cesarean section. Compared with the fixed dose group, the adjusted dose group experienced less spinal blocks to area above the first thoracic dermatome (17.1\% versus $2.2 \%, P=0.022)$ and lower occurrence of hypotension $(50.0 \%$ versus $71.7 \%, P=0.035$ ).

\section{Types of medication}

Each drug or drug class requires a specific dose finding. There are no principal pros and cons to be elaborated. Interestingly, medications within similar therapeutic or structural class may exhibit differences in requirements on weight-based dosing.

\section{Anticoagulants}

LMWHs exhibit difference in requirements on weight-based dosing. Standard dose enoxaparin can be used for chemical prophylaxis of VTE after orthopedic surgery regardless of weight, whereas the dose of nadroparin calcium should be weight-tailored for prevention of VTE after hip replacement surgery. ${ }^{25}$ Regarding direct oral anticoagulants such as apixaban, rivaroxaban, and melagatran, weight-adjusted dosing is unnecessary in the treatment of patients with acute VTE; fixed-dose regimens can be given in the obese population. ${ }^{26-28}$ However, in Japanese patients with nonvalvular atrial fibrillation receiving edoxaban $60 \mathrm{mg}$ once daily, the incidence of all bleeding events in patients $\leq 60 \mathrm{~kg}$ was almost twice that of patients $>60 \mathrm{~kg}$, indicating the necessity of dose reduction in underweight patients $(\leq 60 \mathrm{~kg}){ }^{29}$

\section{Antitumor necrosis factor medications}

Infliximab and adalimumab are both antitumor necrosis factor (anti-TNF) medications. For the treatment of adults 
with moderate-to-severe active Crohn's disease (CD), the recommended intravenous dose of infliximab (REMICADE ${ }^{\circledR}$, Janssen Biotech, Inc., Horsham, PA, USA) is $5 \mathrm{mg} / \mathrm{kg}$ at 0,2 , and 6 weeks, followed by a maintenance dose of $5 \mathrm{mg} / \mathrm{kg}$ every 8 weeks thereafter. ${ }^{30}$ Regarding adalimumab, weight-adjusted dosing is unnecessary. The recommended dose regimen of adalimumab (HUMIRA ${ }^{\circledR}$, AbbVie Inc., North Chicago, IL, USA) for adults with CD is $160 \mathrm{mg}$ initially on the first day, followed by $80 \mathrm{mg}$ on the 15 th day, and $40 \mathrm{mg}$ every other week from the 29th day. ${ }^{31}$ Bhalme et $\mathrm{l}^{32}$ retrospectively analyzed the hospital database of CD patients receiving anti-TNF treatment and determined whether weight-based anti-TNF regimen was favorable. For adalimumab, Kaplan-Meier survival curves indicated that patients with body mass index $(\mathrm{BMI}) \geq 30 \mathrm{~kg} / \mathrm{m}^{2}$ experienced a significantly shorter time to dose escalation $(P=0.01)$, and increases in BMI is associated with an increased hazard of early loss of response $(P=0.04)$. However, there were no significant differences in survival curves for patients with $\mathrm{BMI}<30$ or $\geq 30 \mathrm{~kg} / \mathrm{m}^{2}$ receiving infliximab. BMI is meaningful in anticipating adalimumab efficacy in CD patients, whereas infliximab seems to be advantageous to avoid declining efficacy in obese patients. ${ }^{32}$

\section{P2Y I2-receptor antagonist}

The P2Y12-receptor antagonists (eg, clopidogrel, prasugrel, and ticagrelor) play a crucial role in antiplatelet therapy for patients with myocardial infarction. Payne et $\mathrm{al}^{33}$ identified a negative correlation between clopidogrel effect and body weight ( $r=0.57 ; P=0.002$ ) in patients undergoing carotid surgery, suggesting that weight-tailored dosing may improve clopidogrel therapy. Stable coronary artery disease patients with higher body weight ( $\geq 60 \mathrm{~kg}$ ) had lower plasma concentrations of clopidogrel active metabolite, higher platelet reactivity, and higher on-treatment platelet reactivity rates than patients with low body weight $(<60 \mathrm{~kg}) \cdot{ }^{34}$ Angiolillo et al ${ }^{35}$ observed that BMI could significantly affect the platelet response to clopidogrel loading-dose $(300 \mathrm{mg})$ in patients undergoing coronary stenting $(P=0.04)$. Approximately $59 \%$ of overweight patients (BMI $\geq 25 \mathrm{~kg} / \mathrm{m}^{2}$ ) and $26 \%$ of normal-weight patients $\left(\mathrm{BMI}<25 \mathrm{~kg} / \mathrm{m}^{2}\right.$ ) experienced poor antithrombotic efficacy, indicating that overweight patients may need a higher loading-dose of clopidogrel and/or an adjunct antithrombotic treatment early after the coronary stent procedure. ${ }^{35}$ However, the value of weight-based dosing of clopidogrel needs to be evaluated by prospective studies. Very recently, Olivier et $\mathrm{al}^{36}$ analyzed factors associated with high residual platelet reactivity after administration of third-generation P2Y12-antagonists compared to clopidogrel. In contrast to clopidogrel, platelet reactivity after administration of prasugrel or ticagrelor did not depend on body weight in patients after myocardial infarction, indicating no necessity of increasing doses of prasugrel or ticagrelor to achieve sufficient platelet inhibition in patients with high body weight.

\section{Anti-epidermal growth factor receptor antibody}

Trastuzumab and pertuzumab are monoclonal antibodies for the treatment of human epidermal growth factor receptor 2 (HER2)-positive breast cancer. Current prescribing information specify weight-based dosing method for trastuzumab and fixed dosing for pertuzumab, respectively. ${ }^{37,38}$ Population pharmacokinetic modeling and simulation results showed that the magnitude of body-weight effect on pertuzumab exposure was minimal compared with overall variability and was not clinically relevant, ${ }^{39}$ whereas body weight had a greater effect on interindividual variability of trastuzumab emtansine clearance than other covariates. ${ }^{40}$ Trastuzumab emtansine exposure was relatively consistent across the weight range following body-weight-based dosing. Interestingly, subcutaneous trastuzumab is a ready-to-use liquid formulation that is administered as a $600 \mathrm{mg}$ fixed dose every 3 weeks. The subcutaneous formulation provides the desired pharmacokinetic exposure, with steady-state trough concentrations consistently above the historical efficacy target. This simplifies health care procedures by removing the need for reconstitution and dose calculation according to the body weight of individual. ${ }^{41}$ In the international, randomized, two-cohort PrefHer study, patients with HER2-positive early breast cancer were randomized to receive four adjuvant cycles of $600 \mathrm{mg}$ fixed-dose subcutaneous trastuzumab followed by four cycles of standard intravenous trastuzumab ( $8 \mathrm{mg} / \mathrm{kg}$ loading dose, $6 \mathrm{mg} / \mathrm{kg}$ maintenance doses), or vice versa. Compared with intravenous trastuzumab, subcutaneous trastuzumab has shown noninferior efficacy, similar pharmacokinetic and safety profile, and higher patient preference $(88.9 \%$ versus $9.6 \%, P<0.0001) .{ }^{42,43}$

\section{Weight-based dosing may be superior to currently recommended fixed-dose regimen in adult patients Hydrocortisone}

Hydrocortisone is generally regarded as the preferred replacement therapy for adrenal insufficiency. It is administered at a fixed dose which may be variable and must be individualized 
on the basis of the response of the patient. Mah et $\mathrm{al}^{44}$ examined the variables affecting the pharmacokinetics of hydrocortisone in patients with adrenal insufficiency. Compared with a fixed $10 \mathrm{mg}$ hydrocortisone dose, weightbased dosing could reduce interpatient variability in peak cortisol concentrations in plasma $(7 \%$ versus $31 \%, P<0.05)$ and area under the plasma concentration-time curve $(22 \%$ versus $50 \%, P<0.05)$ and reduce overexposure to steroid from $6.3 \%$ to less than $5 \%$. Body weight was shown as the most distinctive predictor of hydrocortisone clearance, and therefore weight-adjusted dosing is preferred. ${ }^{44}$

\section{Vancomycin}

According to prescribing information, the daily dose of intravenous vancomycin is $2 \mathrm{~g}$ (ie, $500 \mathrm{mg} / 6$ hours or $1 \mathrm{~g} / 12$ hours) for adult patients with normal renal function. However, evidence shows that weight-based dosing is more optimal. Hafermann et $\mathrm{al}^{45}$ identified a preferred vancomycin dosing strategy for patients receiving coronary artery bypass grafting or valve replacement procedures with cardiopulmonary bypass. Weight-based $20 \mathrm{mg} / \mathrm{kg}$ dosing before procedures resulted in vancomycin level being consistently $>15 \mu \mathrm{g} / \mathrm{mL}$ when compared with fixed dosing $(1 \mathrm{~g})$. Catanzano et $\mathrm{al}^{46}$ performed a retrospective analysis of charts on methicillinresistant Staphylococcus aureus (MRSA)-positive patients prior to undergoing elective total joint or spine surgeries. All patients were given $1 \mathrm{~g}$ of vancomycin within an hour prior to surgical incision as prophylaxis. They were assessed as either underdosed (a calculated weight-based dose $>1 \mathrm{~g}$ ) or overdosed (a calculated weight-based dose $<1 \mathrm{~g}$ ) according to the weight-based dosing protocol (15 mg/kg) for vancomycin. Results showed that $69 \%$ of patients were underdosed and $10 \%$ of patients were overdosed. Additionally, the predicted vancomycin level below $15 \mathrm{mg} / \mathrm{L}$ at the end of procedure was observed in $60 \%$ of patients with fixed dose compared to $12 \%$ with weight-based dose $(P=0.0005)$. In order to avoid wrong vancomycin dose to prevent MRSA surgical site infections, physicians must use weight-based dosing rather than fixed dosing. ${ }^{46}$

\section{Linezolid}

According to prescribing information, the dosage of linezolid for adult patients is $600 \mathrm{mg}$ intravenously or orally every 12 hours. Cai et $\mathrm{al}^{47}$ evaluated the drug action of linezolid after a single intravenous fixed dose or a weight-based dose in healthy volunteers. With the $600 \mathrm{mg}$ dose, the lowweight group ( $50 \mathrm{~kg}<$ weight $\leq 55 \mathrm{~kg}$ ) experienced much higher plasma concentrations, serum inhibitory activity, and probability of target attainment compared with high-weight group ( $\geq 80 \mathrm{~kg}$ ). However, this difference was abolished following $10 \mathrm{mg} / \mathrm{kg}$ body-weight dosing. ${ }^{47}$ Hamilton et al ${ }^{48}$ characterized the pharmacokinetics of intravenous and oral linezolid before and after Roux-en-Y gastric bypass surgery and observed that the serum exposure of linezolid was more than 50\% lower in obese compared with nonobese subjects. These data indicate that a weight-tailored dosing of linezolid may be better than fixed dosing for patients of different body weight.

\section{Aprotinin}

Aprotinin is an effective antihemorrhagic during cardiac surgery; in adults, it is usually used regardless of the patient's weight. There was large variation in the aprotinin concentration among patients. A statistically significant correlation was found between aprotinin concentration and patient weight. ${ }^{49}$ Nuttall et $\mathrm{al}^{50}$ observed that a new weight-based dosing schedule could achieve less intrapatient variation in plasma aprotinin concentrations over time compared with the fixed-dose regimen $(P<0.02)$.

\section{Fixed dosing may be noninferior to or even better than currently recommended weight-based regimen in adult patients Cyclosporine microemulsion}

Weight-adjusted cyclosporine for psoriasis treatment is well established. Thaçi et al ${ }^{51}$ investigated the efficacy and safety of fixed dosing (100-300 mg/d) versus weight-based dosing (1.25-5.0 mg/kg/d) of cyclosporine microemulsion in the short-term treatment of severe psoriasis for 12 weeks and concluded that the two dosing methods achieved equal efficacy and safety. In another study, fixed-dosing method of cyclosporine microemulsion seemed feasible in the shortterm treatment of severe atopic dermatitis patients with a body weight of at least $55 \mathrm{~kg}$, and patients over $70 \mathrm{~kg}$ exhibited no difference in efficacy and tolerability compared to those weighing $\leq 70 \mathrm{~kg} .{ }^{52}$

\section{Recombinant activated Factor VII}

Recombinant activated Factor VII (rFVIIa) can be prescribed for treatment of warfarin-associated intracranial hemorrhage (WA-ICH) by rapid normalization of INR. Weight-based dosing method is specified in prescribing information for $\mathrm{rFVIIa}$ (NovoSeven $^{\circledR}$, Novo Nordisk A/S, Bagsværd, Denmark). ${ }^{53}$ Robbins et al ${ }^{54}$ retrospectively compared two rFVIIa hospital protocols for WA-ICH (weight-based dose [80 $\mu \mathrm{g} / \mathrm{kg}$ ] or 
fixed dose [2 mg]). Compared with the weight-based group, the fixed-dose group achieved INR normalization earlier (165 versus 229.5 minutes, $P=0.02$ ) and also had medication acquisition cost savings of $\sim \$ 4,300$ per patient. All other end points were similar between two groups. The low, fixed dose of rFVIIa appeared to be more cost-effective than the high, weight-based dose in achieving INR normalization in patients with WA-ICH. ${ }^{54}$

\section{Epoetin $\alpha$}

Prescribing information for epoetin $\alpha$ requires weightbased dosing method for patients on cancer chemotherapy. A randomized, multicenter study revealed no difference in effectiveness, safety, and quality-of-life benefits of epoetin $\alpha$ in anemic cancer patients on platinum-based chemotherapy between fixed dosing (10,000 IU) and weight-adjusted dosing (150 IU/kg). However, fixed-dose regimen of epoetin $\alpha$ was more convenient than weight-adjusted dose regimen for clinicians and patients. ${ }^{55}$

\section{Ideal body weight-based dosing may be superior to currently recommended total body weight-based regimen in adult patients}

Ideal body weight (IBW) is estimated as follows: IBW $(\mathrm{kg})=50+2.3 \mathrm{~kg}$ for each inch over 5 feet (males); IBW $(\mathrm{kg})=45.5+2.3 \mathrm{~kg}$ for each inch over 5 feet (females). Current prescribing information specify total body weight-based regimen of atracurium and rocuronium. However, evidence showed that IBW-based dosing method was more appropriate for these skeletal muscle relaxants. A randomized study in morbidly obese patients undergoing bariatric surgery showed that IBW-based dosing of atracurium $(0.5 \mathrm{mg} / \mathrm{kg})$ was superior to total body-weight-based dosing $(0.5 \mathrm{mg} / \mathrm{kg})$ in producing enough intubation conditions and regaining muscle strength within 1 hour with no need to use an antagonist. ${ }^{56}$ A study showed that rocuronium dosage should be IBW-based, and total body weight-based dosing of rocuronium significantly prolonged the duration of neuromuscular blockade in morbidly obese patients. ${ }^{57}$ Meyhoff et $\mathrm{al}^{58}$ compared the efficacy of rocuronium for intubation in obese patients receiving gastric banding or gastric bypass when the dose $(0.6 \mathrm{mg} / \mathrm{kg})$ was based upon three weight forms, and concluded that IBW-based dosing could shorten the duration of drug action without significantly prolonging onset time or compromising tracheal intubation conditions compared to corrected body weight-based dosing (IBW $+20 \%$ of excess weight, IBW $+40 \%$ of excess weight).

\section{Dosing in pediatrics \\ Weight-adjusted dosing versus body surface area-adjusted dosing}

Prescribing information for some medications in pediatrics recommend body surface area (BSA)-adjusted dosing method. Methotrexate is usually prescribed based on patient's BSA for children with acute lymphoblastic leukemia (ALL). Jönsson et $\mathrm{al}^{59}$ developed a population pharmacokinetic model in children with ALL. Age, height, body weight, BSA, sex, serum creatinine, and serum alanine aminotransferase were treated as potential covariates. The results showed that weightbased dosing of methotrexate could achieve more predictable pharmacokinetics and better outcome in children with ALL. ${ }^{59}$ Prednisone is recommended for the initial treatment of nephrotic syndrome at a daily dosage of $60 \mathrm{mg} / \mathrm{m}^{2} \mathrm{BSA}$, and a daily dosage of $2 \mathrm{mg} / \mathrm{kg}$ body weight can be prescribed alternatively. A retrospective chart review of children with initial presentation of nephrotic syndrome showed that the daily dose of prednisone at $2 \mathrm{mg} / \mathrm{kg}$ body weight was significantly lower than that at $60 \mathrm{mg} / \mathrm{m}^{2}$ BSA for patients under 30 $\mathrm{kg}(P<0.05)$. However, the difference between two dosing methods narrowed proportionally when patients weighted over $30 \mathrm{~kg} .{ }^{60}$ Very recently, a randomized, open-label, equivalence clinical trial of prednisolone confirmed that clinical outcomes (eg, time taken for remission) with weight-based dosing were equivalent to BSA dosing-related outcomes despite cumulative prednisolone doses being lower in the former and the incidence of hypertension being higher in the BSA group. ${ }^{61}$

Zidovudine and didanosine are nucleoside analogs used for human immunodeficiency virus (HIV) infection. Dosing recommendations of the two drugs should be based on BSA. Weidle et $\mathrm{al}^{62}$ developed a weight-band dosing table to assist appropriate dosing for HIV-infected pediatric patients. Weight-based regimen of zidovudine and didanosine could achieve more accurate dose than BSA-based regimen.

BSA-based dosing of growth hormone could offer noninferiority in efficacy but superior cost-effectiveness in girls with Turner syndrome compared to weight-based dosing. Adult height gain tends to be greater on a BSA-based regimen than on a weight-based regimen in patients who start growth hormone treatment before 8 years of age, and the average financial saving when dosing by BSA was approximately $€ 20,000$ per patient. ${ }^{63}$

Veal et al investigated the adaptive dosing approaches to the individualization of 13-Cis-retinoic acid treatment for children with high-risk neuroblastoma. The medication was administered orally at a daily dose of $160 \mathrm{mg} / \mathrm{m}^{2}$, or 5.33 $\mathrm{mg} / \mathrm{kg}$ for children $<12 \mathrm{~kg}$. Results strongly indicated that a dosage regimen of $5.33 \mathrm{mg} / \mathrm{kg}$ should not be implemented for 
children below $12 \mathrm{~kg}$ because it equated to $24 \%-38 \%$ dose reductions as compared to the standard dose of $160 \mathrm{mg} / \mathrm{m}^{2}$, and $73 \%$ of these young patients failed to achieve the target maximum plasma concentrations. ${ }^{64}$

\section{Weight-adjusted dosing versus age-adjusted manner}

A retrospective study evaluated the clinical outcomes in pediatric patients undergoing hematopoietic stem-cell transplanta tion following weight-based dosing or age-based dosing of intravenous busulfan. Busulfan was given at the dose of $1 \mathrm{mg} / \mathrm{kg}$ for children $<4$ years and $0.8 \mathrm{mg} / \mathrm{kg}$ for children $\geq 4$ years in group 1, whereas busulfan doses were weight-adjusted in group 2 regardless of age. Sinusoidal obstruction syndrome and early infectious complications occurred more frequently in the weight-based dosing group $(\mathrm{HR}=9.46 ; P=0.009)$. The two groups experienced similar occurrence of other chemotherapy complications and overall survival rates. ${ }^{65}$ Therefore, age-based dosing regimen should be suggested when therapeutic drug monitoring of busulfan cannot be performed.

Warad et al ${ }^{66}$ retrospectively analyzed dalteparin's outcomes in the treatment and prophylaxis of VTE in pediatric patients and observed that infants ( $<1$ year) required significantly higher dose to achieve therapeutic anti-Xa levels compared to children (1-10 years) or adolescents (>10-18 years) (396.6 versus 236.7 and $178.8 \mathrm{IU} / \mathrm{kg} / \mathrm{d}$ respectively, $P<0.0001)$. In this single institution experience, dosing was customized in an age-based manner with close monitoring of anti-Xa activity. ${ }^{66}$ This case indicates that the common viewpoint (ie, pediatric patients should be dosed on the basis of body weight) is not always correct.

\section{Weight-based dosing versus pharmacogenetic test-guiding dosing}

In the era of precision medicine, genetic testing for diagnosis and treatment seems to be in great demand. However, pharmacoeconomic issue should not be underestimated. Donnan et $\mathrm{al}^{67}$ assessed the incremental cost-effectiveness per life-month gained in children with ALL receiving 6-mercaptopurine (6-MP) through three dosing strategies, ie, thiopurine methyltransferase (TPMT) genotyping guideddosing, enzymatic testing guided-dosing, and standard weight-adjusted dosing. The enzymatic testing guideddosing and standard weight-adjusted dosing did not show a benefit in survival compared with weight-adjusted dosing. However, the incremental costs per child for genotyping and phenotyping strategy versus weight-adjusted dosing strategy were $\$ 277$ and $\$ 298$, respectively. It does not seem to be cost-effective to conduct pharmacogenetic tests of TPMT before 6-MP initiation in pediatric ALL patients. ${ }^{67}$
Weight-based dosing intervention

Weight estimation, documentation, and dosing determination

Inaccurate weight estimates and omission of weight documentation could potentially contribute to medication dosing errors. A prospective cross-sectional study in West London showed that weight was not documented for $46 \%$ of inpatients and $39 \%$ of inpatients receiving a narrow therapeutic antibiotic due to workflow interruptions and heavy workloads. ${ }^{68}$ Hall et al ${ }^{69}$ examined biases in weight estimation by emergency medical care providers and patients. Patients estimated their own weight more accurately than providers who estimated the patients' weight $(\mathrm{OR}=8.8$, $P=0.00$ ). Providers under 30 years showed more ability to estimate patient weight than older providers. Women's weights were more susceptible to underestimation by providers than men's. ${ }^{69}$ Spicer et $\mathrm{al}^{70}$ assessed the patterns and accuracy of weight estimation in cardiac patients receiving LMWH. Approximately half the patient notes had no record of body weight, and 49\% of LMWH doses were wrong. Heavier patients were more likely to be underestimated, whereas lighter patients were more likely to be overestimated $(P<0.001)$. Nurses were less likely to estimate patient's weight than physicians ( $51 \%$ versus $85 \%, P=0.003)$. Health care providers were found to estimate patient's weight less accurately than patients (39\% versus $80 \%, P<0.001)$, and male patients were more susceptible to inaccurate estimation than female patients ( $44 \%$ versus $62 \%, P=0.035) .{ }^{70}$

Pennsylvania Patient Safety Advisory addresses the significance of accurate patient weights in prevention of medication errors. The organization's policies on medication management and use should ensure that weight-adjusted medications should not be prescribed, dispensed, or administered (except in emergencies) unless patient's weight is available and considered by all relevant health care providers. Prescribers should confirm the accuracy of patient's weight and include the patient's weight on the prescription or physician order prior to weight-based dosing. The calculated dose and the dosing determination such as the dose per weight (eg, $\mathrm{mg} / \mathrm{kg}$ ) should also be specified by the physician; this then becomes beneficial for a pharmacist and/or a nurse to perform an independent double-check of the calculation. ${ }^{71}$

\section{Weight-based dosing chart versus manual calculations}

The application of weight-based dosing chart was proven to be superior to dose estimates derived from manual calculations. The most famous example was weight-based $\mathrm{N}$-acetylcysteine (NAC) regimen for acetaminophen overdose. 
In regard to intravenous infusion, the total dose of NAC is $300 \mathrm{mg} / \mathrm{kg}$ given as three separate doses and administered over a total of 21 hours. The complicated infusion regimen consists of the loading dose of $150 \mathrm{mg} / \mathrm{kg}$, second dose of $50 \mathrm{mg} / \mathrm{kg}$, and third dose of $100 \mathrm{mg} / \mathrm{kg}$, potentially resulting in errors in prescribing, preparing NAC infusions, and administration in ED patients. Errors made by physicians and nurses due to manual calculations were observed with $26 \%$ of cases, whereas the use of weight-based dosing chart showed $100 \%$ accuracy in dose calculations and improved patient safety. ${ }^{72}$ Another study confirmed the role of weight-based dosing chart to decrease NAC prescription errors associated with diluent (50.6\% versus $4.8 \%, P<0.001)$, dose $(13.6 \%$ versus $0.0 \%, P=0.01)$, and drip rate $(11.1 \%$ versus $0.0 \%$, $P=0.03){ }^{73}$

Dosing protocol, order set, pharmacist participation, technological information, and educational measures

Vancomycin dosing regimen (15-20 mg/kg every 8-12 hours) is recommended for patients with normal renal function. A weight-based dosing intervention study of vancomycin showed that dosing protocol could improve the initial dosing and enable more patients rapidly achieve therapeutic concentration. After intervention, patients received higher median initial doses (20 versus $12.5 \mathrm{mg} / \mathrm{kg} ; P<0.001$ ) and larger median value of area under the plasma concentration time curve (366.0 versus $262.5 \mathrm{mg} \cdot \mathrm{h} / \mathrm{L} ; P<0.01) .{ }^{74}$ Marquis et $\mathrm{al}^{75}$ evaluated the effect of a pharmacist-led program of vancomycin dosing and monitoring on the proportion of patients receiving weight-adjusted dosing regimen. Pharmacistled interventions resulted in more than half the patients being dosed appropriately $(P<0.001)$, a shorter duration of treatment (10.0 versus 8.4 days, $P<0.003$ ), and a lower incidence of nephrotoxicity $(8.7 \%$ versus $3.2 \%, P=0.006) .{ }^{75}$ A retrospective study showed that electronic weight-based vancomycin order set integrated with computer physician order entry could significantly increase the percentage of appropriate dosing in ED patients (67.4\% versus $45.5 \%$, $P<0.05){ }^{76}$

Russell et $\mathrm{al}^{77}$ described a hospital protocol utilizing automatic dosage adjustments and pharmacist consultations to optimize the use of eight antimicrobials and two anticoagulant agents in obese patients. Evidence-based recommendations on initial dosing based upon weight and renal function were formulated. Under the protocol, pharmacists would receive electronic alerts regarding protocol-eligible patients during initial order verification and automatically adjust medication dosages as appropriate. For patients prescribed anticoagulants at specified dose levels, clinical pharmacists would consult with prescribers to help ensure safe and effective initial and ongoing therapy. Multidisciplinary educational initiatives were conducted before protocol implementation. Pharmacists implemented weight- and renal function-based dosage adjustments for obese patients achieved $89 \%$ compliance with the protocol and no documented adverse drug reactions. ${ }^{77}$

Aurora Health Care established an integrated health care system to reduce the overall time to achieving effective anticoagulation. The system created an intervention primarily consisting of a preprinted order sheet, a weightadjusted heparin dosing nomogram, and an education plan for physicians and other health care professionals. Overall rates of adequate anticoagulation within the first 24 hours was significantly improved from $73 \%$ to $95 \%{ }^{78}$

\section{Further research opportunities}

First, it is worthwhile to go for a much more specific systematic analysis of weight-based dosing in pediatrics. Second, numerous studies should aim to investigate the factors determining the applicability of weight-tailored dosing in clinical practice, especially in clinical circumstance in which dose-effect relationship seems complicated. Third, for some medications, especially high-alert medications, expensive medications, and antibacterials, it is necessary to compare different dosing methods (ie, total body weight-based dosing versus fixed dosing, IBW-based dosing, BSA-based dosing, age-based dosing, or pharmacogenetic test-guiding dosing) from the perspectives of efficacy, safety, pharmacoeconomics, patient preference, and adherence. Fourth, population pharmacokinetic study should be encouraged because it can effectively screen the contribution of body weight to drug clearance. Fifth, it is useful to evaluate body weight's effect on dosage by performing retrospective chart review in combination with statistical analysis such as multivariate stepwise linear regression analysis and Cox proportional hazards model. If possible, a prospective clinical trial can be designed in the future. Finally, it is necessary to strengthen weight-based dosing intervention studies and evaluate the cost-effectiveness of a comprehensive and integrated program including motivational, information technological, educational, and organizational measures.

\section{Conclusion}

Although each drug or drug class requires a specific dose finding, there are no principal pros and cons to be elaborated. This review of weight-based dosing strategy will enrich the knowledge of safety, efficacy, and economics-oriented medication administration as well as help in finding research opportunities in clinical practice. Clinicians should be 
familiar with dosage and administration of the medication to be prescribed as well as the latest developments.

\section{Acknowledgments}

This work was supported by Zhejiang Provincial Bureau of Education (grant N20140209) and National Natural Science Foundation of China (grant 81373488). The authors would also like to thank Professor Xiao-na Dai, Professor Mei-juan Lan, and Professor Xiao-ying Zhao for their kind help in medication management and use.

\section{Disclosure}

The authors report no conflicts of interest in this work.

\section{References}

1. Joint Commission Resources. Joint Commission International Accreditation Standards for Hospitals. 5th ed. Oak Brook, IL: Joint Commission Resources; 2013.

2. ECRI Institute. Medication safety: inaccurate patient weight can cause dosing errors. Available from: https:/www.ecri.org/components/ PSOCore/Pages/PSONav0214.aspx. [updated February 1, 2014]. Accessed December 19, 2015.

3. Masanja IM, Selemani M, Khatib RA, et al. Correct dosing of artemether-lumefantrine for management of uncomplicated malaria in rural Tanzania: do facility and patient characteristics matter? Malar J. 2013;12:446.

4. Rosini JM, Grovola MR, Levine BJ, Jasani NB. Prescribing habits of vancomycin in the Emergency Department: are we dosing appropriately? J Emerg Med. 2013;44:979-984.

5. Ritchie DJ, Reichley RM, Canaday KL, Bailey TC. Evaluation and financial impact of imipenem/cilastatin dosing in elderly patients based on renal function and body weight. J Pharm Technol. 1993;9:160.

6. Gibson PS, Newell K, Sam DX, et al. Weight-adjusted dosing of tinzaparin in pregnancy. Thromb Res. 2013;131:e71-e75.

7. Luscombe DK. Factors influencing plasma drug concentrations. J Int Med Res. 1977;5(1 Suppl):82-97.

8. Hodkinson JP, Lucas M, Lee M, Harrison M, Lunn MP, Chapel H. Therapeutic immunoglobulin should be dosed by clinical outcome rather than by body weight in obese patients. Clin Exp Immunol. 2015;181:179-187.

9. drugs.com [homepage on the Internet]. Nadroparin calcium (Fraxiparine) [updated March 21, 2001]. Available from: http://www.drugs.com/cons/ fraxiparine.html. Accessed December 19, 2015.

10. RxList [homepage on the Internet]. Enoxaparin (Lovenox) [updated July 16, 2013]. Available from: http://www.rxlist.com/lovenox-drug/ indications-dosage.htm. Accessed December 19, 2015.

11. Priglinger U, Delle Karth G, Geppert A, et al. Prophylactic anticoagulation with enoxaparin: is the subcutaneous route appropriate in the critically ill? Crit Care Med. 2003;31:1405-1409.

12. Nunez JM, Becher RD, Rebo GJ, et al. Prospective evaluation of weightbased prophylactic enoxaparin dosing in critically ill trauma patients: adequacy of AntiXa levels is improved. Am Surg. 2015;81:605-609.

13. Chapman SA, Irwin ED, Reicks P, Beilman GJ. Non-weight-based enoxaparin dosing subtherapeutic in trauma patients. J Surg Res. 2016; 201:181-187.

14. RxList [hompage on the Internet]. ZYVOX ${ }^{\circledR}$ [updated June 23, 2015]. Available from: http://www.rxlist.com/zyvox-drug/indications-dosage. htm. Accessed December 19, 2015.

15. Niwa T, Watanabe T, Suzuki A, et al. Reduction of linezolid-associated thrombocytopenia by the dose adjustment based on the risk factors such as basal platelet count and body weight. Diagn Microbiol Infect Dis. 2014;79:93-97.
16. Maki N, Ohkuchi A, Tashiro $\mathrm{Y}$, et al. Initial dose of vancomycin based on body weight and creatinine clearance to minimize inadequate trough levels in Japanese adults. Eur J Clin Microbiol Infect Dis. 2012;31:2537-2543.

17. RxList [hompage on the Internet]. Zemplar [updated May 20, 2011]. Available from: http://www.rxlist.com/zemplar-drug/indicationsdosage.htm. Accessed December 19, 2015.

18. Martin KJ, González E, Lindberg JS, et al. Paricalcitol dosing according to body weight or severity of hyperparathyroidism: a double-blind, multicenter, randomized study. Am J Kidney Dis. 2001;38:S57-S63.

19. Miao L, Yang J, Huang C, Shen Z. Contribution of age, body weight, and CYP2C9 and VKORC1 genotype to the anticoagulant response to warfarin: proposal for a new dosing regimen in Chinese patients. Eur J Clin Pharmacol. 2007;63:1135-1141.

20. Yoo SH, Nah HW, Jo MW, et al. Age and body weight adjusted warfarin initiation program for ischaemic stroke patients. Eur J Neurol. 2009; 16:1100-1105.

21. Yoo SH, Kwon SU, Jo MW, Kang DW, Kim JS. Age- and weightadjusted warfarin initiation nomogram for ischaemic stroke patients. Eur J Neurol. 2012;19:1547-1553.

22. Jung JA, Kim TE, Lee H, et al. A proposal for an individualized pharmacogenetic-guided isoniazid dosage regimen for patients with tuberculosis. Drug Des Devel Ther. 2015;9:5433-5438.

23. Subedi A, Tripathi M, Bhattarai BK, Gupta PK, Pokharel K, Regmi MC. The effect of height and weight adjusted dose of intrathecal hyperbaric bupivacaine for elective caesarean section. JNMA J Nepal Med Assoc. 2011;51:1-6.

24. Harten JM, Boyne I, Hannah P, Varveris D, Brown A. Effects of a height and weight adjusted dose of local anaesthetic for spinal anaesthesia for elective Caesarean section. Anaesthesia. 2005;60:348-353.

25. Kovacs MJ, Weir K, MacKinnon K, Keeney M, Brien WF, Cruickshank MK. Body weight does not predict for anti-Xa levels after fixed dose prophylaxis with enoxaparin after orthopedic surgery. Thromb Res. 1998;91:137-142.

26. Sarich TC, Teng R, Peters GR, et al. No influence of obesity on the pharmacokinetics and pharmacodynamics of melagatran, the active form of the oral direct thrombin inhibitor ximelagatran. Clin Pharmacokinet. 2003;42:485-492.

27. Upreti VV, Wang J, Barrett YC, et al. Effect of extremes of body weight on the pharmacokinetics, pharmacodynamics, safety and tolerability of apixaban in healthy subjects. Br J Clin Pharmacol. 2013;76: 908-916.

28. Kubitza D, Becka M, Zuehlsdorf M, Mueck W. Body weight has limited influence on the safety, tolerability, pharmacokinetics, or pharmacodynamics of rivaroxaban (BAY 59-7939) in healthy subjects. $J$ Clin Pharmacol. 2007;47:218-226.

29. Yamashita T, Koretsune Y, Yasaka M, et al. Randomized, multicenter, warfarin-controlled phase II study of edoxaban in Japanese patients with non-valvular atrial fibrillation. Circ J. 2012;76:1840-1847.

30. RxList [homepage on the Internet]. Remicade [updated October 9, 2015]. Available from: http://www.rxlist.com/remicade-drug.htm. Accessed December 19, 2015.

31. RxList [homepage on the Internet]. Humira [updated September 23, 2015]. Available from: http://www.rxlist.com/humira-drug.htm. Accessed December 19, 2015.

32. Bhalme M, Sharma A, Keld R, Willert R, Campbell S. Does weight-adjusted anti-tumour necrosis factor treatment favour obese patients with Crohn's disease? Eur J Gastroenterol Hepatol. 2013;25:543-549.

33. Payne DA, Jones CI, Hayes PD, Naylor AR, Goodall AH. Therapeutic benefit of low-dose clopidogrel in patients undergoing carotid surgery is linked to variability in the platelet adenosine diphosphate response and patients' weight. Stroke. 2007;38:2464-2469.

34. Wagner H, Angiolillo DJ, Ten Berg JM, et al. Higher body weight patients on clopidogrel maintenance therapy have lower active metabolite concentrations, lower levels of platelet inhibition, and higher rates of poor responders than low body weight patients. J Thromb Thrombolysis. 2014;38:127-136. 
35. Angiolillo DJ, Fernández-Ortiz A, Bernardo E, et al. Platelet aggregation according to body mass index in patients undergoing coronary stenting: should clopidogrel loading-dose be weight adjusted? J Invasive Cardiol. 2004;16:169-174.

36. Olivier CB, Schnabel K, Weber S, et al. Platelet reactivity after administration of third generation P2Y12-antagonists does not depend on body weight in contrast to clopidogrel. J Thromb Thrombolysis. Epub February 2, 2016.

37. RxList [homepage on the Internet]. Herceptin [updated May 14, 2015]. Available from: http://www.rxlist.com/herceptin-drug/indicationsdosage.htm. Accessed February 19, 2016.

38. RxList [homepage on the Internet]. Perjeta [updated June 12, 2015] Available from: http://www.rxlist.com/perjeta-drug/indications-dosage htm. Accessed February 19, 2016

39. Garg A, Quartino A, Li J, et al. Population pharmacokinetic and covariate analysis of pertuzumab, a HER2-targeted monoclonal antibody, and evaluation of a fixed, non-weight-based dose in patients with a variety of solid tumors. Cancer Chemother Pharmacol. 2014;74:819-829.

40. Gupta M, Lorusso PM, Wang B, et al. Clinical implications of pathophysiological and demographic covariates on the population pharmacokinetics of trastuzumab emtansine, a HER2-targeted antibodydrug conjugate, in patients with HER2-positive metastatic breast cancer. J Clin Pharmacol. 2012;52:691-703.

41. Quartino AL, Hillenbach C, Li J, et al. Population pharmacokinetic and exposure-response analysis for trastuzumab administered using a subcutaneous "manual syringe" injection or intravenously in women with HER2-positive early breast cancer. Cancer Chemother Pharmacol. 2016;77:77-88.

42. Pivot X, Gligorov J, Müller V, et al. Patients' preferences for subcutaneous trastuzumab versus conventional intravenous infusion for the adjuvant treatment of HER2-positive early breast cancer: final analysis of 488 patients in the international, randomized, two-cohort PrefHer study. Ann Oncol. 2014;25:1979-1987.

43. Jin JF, Zhu LL, Chen M, et al. The optimal choice of medication administration route regarding intravenous, intramuscular, and subcutaneous injection. Patient Prefer Adherence. 2015;9:923-942.

44. Mah PM, Jenkins RC, Rostami-Hodjegan A, et al. Weight-related dosing, timing and monitoring hydrocortisone replacement therapy in patients with adrenal insufficiency. Clin Endocrinol (Oxf). 2004;61:367-375.

45. Hafermann MJ, Kiser TH, Lyda C, et al. Weight-based versus set dosing of vancomycin for coronary artery bypass grafting or aortic valve surgery. J Thorac Cardiovasc Surg. 2014;147:1925-1930.

46. Catanzano A, Phillips M, Dubrovskaya Y, Hutzler L, Bosco J 3rd. The standard one gram dose of vancomycin is not adequate prophylaxis for MRSA. Iowa Orthop J. 2014;34:111-117.

47. Cai Y, Chai D, Falagas ME, et al. Weight-adjusted versus fixed dose of linezolid for Chinese healthy volunteers of higher and lower body weight: a Phase I pharmacokinetic and pharmacodynamic study. Expert Opin Investig Drugs. 2013;22:309-315.

48. Hamilton R, Thai XC, Ameri D, Pai MP. Oral bioavailability of linezolid before and after Roux-en-Y gastric bypass surgery: is dose modification necessary in obese subjects? J Antimicrob Chemother. 2013;68: 666-673.

49. Beath SM, Nuttall GA, Fass DN, Oliver WC Jr, Ereth MH, Oyen LJ. Plasma aprotinin concentrations during cardiac surgery: full- versus half-dose regimens. Anesth Analg. 2000;91:257-264.

50. Nuttall GA, Fass DN, Oyen LJ, Oliver WC Jr, Ereth MH. A study of a weight-adjusted aprotinin dosing schedule during cardiac surgery. Anesth Analg. 2002;94:283-289.

51. Thaçi D, Bräutigam M, Kaufmann R, Weidinger G, Paul C, Christophers E. Body-weight-independent dosing of cyclosporine micro-emulsion and three times weekly maintenance regimen in severe psoriasis. A randomised study. Dermatology. 2002;205:383-388.

52. Czech W, Bräutigam M, Weidinger G, Schöpf E. A body-weightindependent dosing regimen of cyclosporine microemulsion is effective in severe atopic dermatitis and improves the quality of life. $J$ Am Acad Dermatol. 2000;42:653-659.
53. RxList [homepage on the Internet]. NovoSeven ${ }^{\circledR}$ [updated January 12, 2009]. Available from: http://www.rxlist.com/novosevendrug/indications-dosage.htm. Accessed December 19, 2015.

54. Robbins A, Fong J, Hall W, Ditch K, Rolfe S, Miller M. Comparison of a low, fixed dose and a high, weight-based dose of recombinant factor VIIa in the treatment of warfarin-associated intracranial hemorrhage. Neurocrit Care. 2014;20:466-469.

55. Granetto C, Ricci S, Martoni A, et al. Comparing the efficacy and safety of fixed versus weight-based dosing of epoetin alpha in anemic cancer patients receiving platinum-based chemotherapy. Oncol Rep. 2003; 10:1289-1296.

56. van Kralingen S, van de Garde EM, Knibbe CA, et al. Comparative evaluation of atracurium dosed on ideal body weight vs total body weight in morbidly obese patients. Br J Clin Pharmacol. 2011;71: 34-40.

57. Leykin Y, Pellis T, Lucca M, Lomangino G, Marzano B, Gullo A. The pharmacodynamic effects of rocuronium when dosed according to real body weight or ideal body weight in morbidly obese patients. Anesth Analg. 2004;99:1086-1089.

58. Meyhoff CS, Lund J, Jenstrup MT, et al. Should dosing of rocuronium in obese patients be based on ideal or corrected body weight? Anesth Analg. 2009;109:787-792.

59. Jönsson P, Skärby T, Heldrup J, Schrøder H, Höglund P. High dose methotrexate treatment in children with acute lymphoblastic leukaemia may be optimised by a weight-based dose calculation. Pediatr Blood Cancer. 2011;57:41-46.

60. Feber J, Al-Matrafi J, Farhadi E, Vaillancourt R, Wolfish N. Prednisone dosing per body weight or body surface area in children with nephrotic syndrome: is it equivalent? Pediatr Nephrol. 2009;24: 1027-1031.

61. Raman V, Krishnamurthy S, Harichandrakumar KT. Body weight-based prednisolone versus body surface area-based prednisolone regimen for induction of remission in children with nephrotic syndrome: a randomized, open-label, equivalence clinical trial. Pediatr Nephrol. 2016;31: 595-604.

62. Weidle PJ, Abrams EJ, Gvetadze R, Rivadeneira E, Kline MW. A simplified weight-based method for pediatric drug dosing for zidovudine and didanosine in resource-limited settings. Pediatr Infect Dis J. 2006;25: 59-64.

63. Schrier L, de Kam ML, McKinnon R, et al. Comparison of body surface area versus weight-based growth hormone dosing for girls with Turner syndrome. Horm Res Paediatr. 2014;81:319-330.

64. Veal GJ, Errington J, Rowbotham SE, et al. Adaptive dosing approaches to the individualization of 13-cis-retinoic acid (isotretinoin) treatment for children with high-risk neuroblastoma. Clin Cancer Res. 2013;19: 469-479.

65. Gürlek Gökçebay D, Azik F, Ozbek N, et al. Clinical comparison of weight- and age-based strategy of dose administration in children receiving intravenous busulfan for hematopoietic stem cell transplantation. Pediatr Transplant. 2015;19:307-315.

66. Warad D, Rao AN, Mullikin T, et al. A retrospective analysis of outcomes of dalteparin use in pediatric patients: a single institution experience. Thromb Res. 2015;136:229-233.

67. Donnan JR, Ungar WJ, Mathews M, Hancock-Howard RL, Rahman P. A cost effectiveness analysis of thiopurine methyltransferase testing for guiding 6-mercaptopurine dosing in children with acute lymphoblastic leukemia. Pediatr Blood Cancer. 2011;57:231-239.

68. Charani E, Gharbi M, Hickson M, et al. Lack of weight recording in patients being administered narrow therapeutic index antibiotics: a prospective cross-sectional study. BMJ Open. 2015;5:e006092.

69. Hall WL 2nd, Larkin GL, Trujillo MJ, Hinds JL, Delaney KA. Errors in weight estimation in the emergency department: comparing performance by providers and patients. J Emerg Med. 2004;27: 219-224.

70. Spicer K, Gibson P, Bloe C, Cross SJ, Leslie SJ. Weight assessment in cardiac patients: implications for prescription of low molecular weight heparin. Postgrad Med J. 2009;85:124-127. 
71. Pennsylvania Patient Safety Advisory. medication errors: significance of accurate patient weights. Pa Patient Saf Advis. 2009;6(1):10-15. Available from: http://patientsafetyauthority.org/ADVISORIES/ AdvisoryLibrary/2009/mar6(1)/Pages/10.aspx. Accessed March 17, 2016.

72. Selvan VA, Calvert SH, Cavell G, Glucksman E, Kerins M, Gonzalez J. Weight-based N-acetylcysteine dosing chart to minimise the risk of calculation errors in prescribing and preparing $\mathrm{N}$-acetylcysteine infusions for adults presenting with paracetamol overdose in the emergency department. Emerg Med J. 2007;24:482-484.

73. McIntyre S, McD Taylor D, Greene S. Introduction of an N-acetylcysteine weight-based dosing chart reduces prescription errors in the treatment of paracetamol poisoning. Emerg Med Australas. 2013;25: 28-35.

74. Li J, Udy AA, Kirkpatrick CM, Lipman J, Roberts JA. Improving vancomycin prescription in critical illness through a drug use evaluation process: a weight-based dosing intervention study. Int $J$ Antimicrob Agents. 2012;39:69-72.
75. Marquis KA, DeGrado JR, Labonville S, Kubiak DW, Szumita PM. Evaluation of a pharmacist-directed vancomycin dosing and monitoring pilot program at a tertiary academic medical center. Ann Pharmacother. 2015;49:1009-1014.

76. Hall AB, Montero J, Cobian J, Regan T. The effects of an electronic order set on vancomycin dosing in the ED. Am J Emerg Med. 2015;33:92-94.

77. Russell JM, Nick-Dart RL, Nornhold BD. Development of a pharmacistdriven protocol for automatic medication dosage adjustments in obese patients. Am J Health Syst Pharm. 2015;72:1656-1663.

78. Berry BB, Geary DL, Jaff MR. A model for collaboration in quality improvement projects: implementing a weight-based heparin dosing nomogram across an integrated health care delivery system. Jt Comm J Qual Improv. 1998;24:459-469.

\section{Publish your work in this journal}

Patient Preference and Adherence is an international, peer-reviewed, open access journal that focuses on the growing importance of patient preference and adherence throughout the therapeutic continuum. Patient satisfaction, acceptability, quality of life, compliance, persistence and their role in developing new therapeutic modalities and compounds to optimize clinical outcomes for existing disease states are major areas of interest for the journal. This journal has been accepted for indexing on PubMed Central. The manuscript management system is completely online and includes a very quick and fair peer-review system, which is all easy to use. Visit http://www dovepress.com/testimonials.php to read real quotes from published authors.

Submit your manuscript here: http://www.dovepress.com/patient-preference-and-adherence-journal 